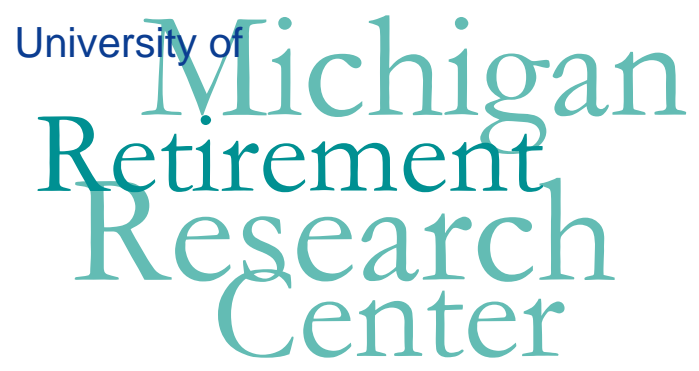

Working Paper

WP 2009-217

\title{
Marking Social Security's Open Group Liability to Market
}

\author{
Paul A. Koehler and Laurence J. Kotlikoff
}

\begin{tabular}{|l|l|l|}
\hline $\mathrm{M}$ & $\mathrm{R}$ \\
\hline $\mathrm{R}$ & $\mathrm{C}$ \\
\hline
\end{tabular}$\quad$ Project \#: UM09-03 



\title{
Marking Social Security’s Open Group Liability to Market
}

\author{
Paul A. Koehler \\ Boston University \\ Laurence J. Kotlikoff \\ Boston University \\ September 2009 \\ Michigan Retirement Research Center \\ University of Michigan \\ P.O. Box 1248 \\ Ann Arbor, MI 48104 \\ http://www.mrrc.isr.umich.edu/ \\ (734) 615-0422
}

\section{Acknowledgements}

This work was supported by a grant from the Social Security Administration through the Michigan Retirement Research Center (Grant \# 10-M-98362-5-01). The findings and conclusions expressed are solely those of the author and do not represent the views of the Social Security Administration, any agency of the Federal government, or the Michigan Retirement Research Center.

\section{Regents of the University of Michigan}

Julia Donovan Darrow, Ann Arbor; Laurence B. Deitch, Bingham Farms; Denise Ilitch, Bingham Farms; Olivia P. Maynard, Goodrich; Andrea Fischer Newman, Ann Arbor; Andrew C. Richner, Grosse Pointe Park; S. Martin Taylor, Gross Pointe Farms; Katherine E. White, Ann Arbor; Mary Sue Coleman, ex officio 


\title{
Marking Social Security’s Open Group Liability to Market
}

\begin{abstract}
This paper marks Social Security's open group liability to market taking into account the riskiness of its aggregate benefit payments and tax receipts. The open group liability references the present value of the system's net cash flow from now through the indefinite future. We treat the growth rates of the system's aggregate benefits and taxes as implicit securities that are spanned by the returns on marketed securities. Our pricing of Social Security's infinite horizon net liability builds on prior independent work by Blocker, Kotlikoff, and Ross (2009) and Geanakoplos and Zeldes (2009). Our results, which we view as preliminary, suggest that the market value of Social Security's open group liability may be many times larger than the \$15.1 trillion stated in the Trustees' Report. Unlike Blocker, Kotlikoff, and Ross (2009), this discrepancy between our financial valuation and Social Security's does not reflect differences in the value assumed for the safe rate of return. To control for this factor, we simply follow Social Security (and Geanakoplos, et. al., 2009) in assuming a 2.9 percent safe real rate of discount. We also find that the precise marketed assets used to price future Social Security benefits and taxes can significantly alter the estimate of the open group liability.
\end{abstract}




\section{Introduction}

There are many ways to measure the health of the U.S. Social Security system. In particular, one can measure what it will cost to keep the system operating as is and what it would cost to immediately shut the system down with respect to any additional tax payments or benefit accruals. The former measure is called Social Security's open group liability. The later measure is called Social Security accrued liability (alternatively, "shut down cost" or "maximium transition cost", where transition references moving to a privatized system).

This paper considers the system's open group liability, but unlike the valuation procedures used by the system's actuaries and reported in the annual Trustees Report, our analysis adjusts for the riskiness of future benefit payments and tax receipts. This is surely a plus relative to the current state of the valuation art, but it foregoes many of the microeconomic details used in Social Security's benefit and tax projections.

Our method involves treating aggregate Social Security benefit payments and tax receipts as implicit financial securities. Specifically, we model the growth rate of these aggregates as implicit financial assets whose returns are spanned by the current set of marketed securities.

In its January 1, 2009 the annual Social Security Administration (SSA) Trustees Report, the Trustees put the present value of Social Security's open group Old Age Survivors Disability Insurance (OASDI) unfunded liability at $\$ 15.1$ trillion $^{2}$. To arrive at its estimate, SSA makes benefit and contribution projections by type of benefit and contribution for various cohorts and groups within cohorts. To this end, Social Security selects low, intermediate, and high growth rates of fertility, mortality, real wages, etc. Then, for each set of assumptions, Social Security treats future growth rates as sure things and discounts at the assumed safe rate of return.

The valuation methods proposed in this paper abstract from the determinants of demographic and individual benefit accrual. Instead, we focus on only two growth rates, those of

\footnotetext{
${ }^{2}$ SSA 2009 Trustees Report is available at http://www.ssa.gov/OACT/TR/2009/trTOC.html
} 
aggregate OASDI benefits and aggregate OASDI contributions. We then suppose that there exists securities whose payoffs can be used to replicate the same return as the growth rate security up to an idiosyncratic error (e.g., measurement error) that is not valued in the market. Next we determine what financial markets would pay for these securities by projecting the payoff growth rates onto a set of returns for traded assets, i.e. by using simple ordinary least squares (OLS) regressions to detect the set of spanning assets.

We find that the Social Security Administration (SSA) may be underestimating the size of its open group unfunded liability by a factor of four to five. However, our results are preliminary and highly sensitive to assumptions, including the set of spanning assets. In particular, the inclusion of longer-term bond returns seems to destabilize the valuation of the growth rate securities.

The remainder of this paper is organized as follows. The next section provides a review of two prior studies, Blocker, Kotlikoff, and Ross (2009), henceforth BKR, and Geanakoplos and Zeldes (2009), henceforth GZ, on which this paper builds. Section three details the methodology used to risk-adjust the open group net liability. Section four presents and discusses findings. The last section summarizes key results and discusses the limitations of the methodology employed as well as our plans for future work on the topic.

\section{Literature Review}

The two studies to which this paper most directly relate are those of BKR and GZ. The BKR piece is of particular interest because this paper's methodology draws heavily on their approach. In their paper, BKR attempt to value SSA's net operating liability to workingage Americans rather than the accrued liability to that populaton. BKR focus solely on OAI retirement benefits and OASI taxes used to finance those benefits. BKR report a net OAI liability to current working-age Americans of $\$ 10.4$ trillion, which is approximately $23 \%$ larger 
than the corresponding liability calculated using SSA's intermediate constant wage-growth rate and discount rate assumptions.

BKR show that the formula for a worker's Primary Insurance Amount (PIA) can be decomposed into the product of a) an idiosyncratic component, which is a function of the average value across a worker's workspan of the ratio of her covered earnings in a given year to the economy-wide average wage in that year and b) a market component whose value depends on how the financial markets implicitly value average wage growth.

BKR specify a random effects model for relative wages over the work span and use it to estimate the idiosyncratic component of workers' PIAs. The data used in this analysis come from the Panel Study of Income Dynamics. BKR also show that the market component of the PIA (the component of the PIA that is assumed to be priced by the market) corresponds to a wage-growth security. For a worker born in year $\tau$, this market component or factor can be written as the product of the current average wage, $\bar{w}_{t}$ (where $t \geq \tau$ ), multiplied by cumulated (through age 60) future wage growth rate factors, i.e.,

$$
\bar{w}_{\tau+60}=\bar{w}_{t} \prod_{k=t+1}^{\tau+60}\left(1+g_{k}\right)
$$

where $g_{k}$ is the growth rate of the real average wage in period $k$.

While SSA assumes a constant average wage growth rate, implying that all parts of (1) are known at time $t$, BKR argue that such an assumption is at odds with the true wage-growth rate process which, they show, is actually quite noisy. Therefore, they maintain the stochastic growth rate assumption. As such, the product of the gross wage-growth rate factors in (1) is the uncertain quantity of interest.

To see why this quantity represents the return from a wage-growth security, suppose an individual holds $\$ 1$ worth of the S\&P 500 stock index at time $k$. Then the individual's portfolio in one year is worth $\$ 1 \cdot\left(1+r_{k}\right)$ where $r_{k}$ is the net rate of return on the S\&P 500 
over that holding period. If the individual holds this portfolio for two periods, then his return is $\$ 1 \cdot\left(1+r_{k}\right)\left(1+r_{k+1}\right)$. Clearly the return for the generalized $T$-period horizon takes the very same form as the product of the wage-growth rates. Therefore, BKR suggest considering a hypothetical security which pays not the return on the S\&P 500, but an annual rate of return equal to the annual growth rate in average real wages.

Interpreting the product of the wage-growth rate factors as the payoff of a financial security leads naturally to the question: How much would the market pay for $\$ 1$ of this security. Note that such a security does not currently exist in financial markets. Therefore, BKR project the wage growth rates onto the space spanned by the returns of regularly traded assets. Specifically, BKR regress aggregate wage-growth rates on a constant and a set of $n$ asset returns

$$
g_{t}=\alpha+\sum_{i=1}^{n} \beta_{i} s_{i, t}+\epsilon_{t}
$$

where $s_{i, t}$ is the return on asset $i$ for period $t$ and $\epsilon_{t}$ is an idiosyncratic error term that is uncorrelated with asset returns. Arbitrage Pricing Theory (APT) then suggests that the price of the wage growth security equals the value of the replicating portfolio of traded assets, assuming that the replicating portfolio captures and prices all of the non-idiosyncratic return characteristics of the wage-growth rate. They also run a similar regression except using one-period lags of the asset returns.

To see how one goes from these coefficients to valuing wage growth securities, rewrite regression (2) in the following manner:

$$
1+g_{t}=\delta(1+r)+\sum_{i=1}^{n} \beta_{i}\left(1+s_{i, t}\right)+\epsilon_{t},
$$

where $\mathrm{r}$ is the safe rate of return and $\delta$ is defined to make the two versions of the regressions consistent. In this format, it's easy to see that investing 1 dollar for one period in the wage 
growth security will yield, except for an assumed valueless error, the same return as investing $\delta$ in the safe asset and $\beta_{i}$ in risky asset $i$. So the sum of $\delta$ and the $\beta_{i}$ s is the total value of the 1 dollar wage growth security held for one period. Let's suppose this value is 79 cents; i.e., investing 1 dollar for one period in the wage growth security is equivalent to investing 79 cents in safe and risky assets.

But why would anyone want to invest 1 when doing so in the required manner is only worth 79? The answer is because they are forced to. Social Security isn't a voluntary system. We have to pay the taxes for which we are liable, and we are also forced to accrue the benefits the system provides conditional on what we earn. Incidentally, there is nothing that requires that 1 invested in the wage-growth security for one period be worth less than 1 . It could be worth more than 1 depending on the size of the coefficients, which are capturing how the security's mean (how it will preform on average) and its co-movement with the risky marketed assets.

So how much is a two-period wage-growth security worth, where again, the security exists and is being held by its owner, at least initially? I.e., what's the market value of being told that someone (Uncle Sam, for example) has invested for you 1 dollar today and he will pay you at the end of two periods the 1 compounded for two periods at the prevailing wage-growth rate? The answer is 79 cents $x 79$ cents. The reason is that this security must, by arbitrage, have the same value as cashing it out today for 79 cent, investing it in the safe asset and thus ending up a period from now with 79 cents times $(1+r)$ and then investing/being forced to invest that amount in the one-period wage-growth security that is worth 79 cents for each dollar invested. So the original investment translates into 79 cents times $(1+r)$ times 79 cents. But discounted back to today, that's simply 79 cents squared. This arbitrage reasoning carries through to any holding period, so the value of an $n$-period wage-growth security is 79 cents raised to the power $n$. BKR view each future year's tax payments and benefits receipts as involving, apart from the idiosyncratic component, a wage growth security of a specific maturity; i.e., OASDI taxes to be paid 15 years from the present involve a 15-year wage-growth security because the holding 
period on the security is 15 years.

Armed with estimates of the idiosyncratic and market valuation components of a worker's benefits, BKR then calculate the present value of the aggregate benefits currently owed to working-age Americans. Through a similar process they value the aggregate taxes. The difference between these two valuations produces a net operating liability in real 2005 dollars of \$10.4 trillion, where, again, the term "operating" references the assumption that Social Security's tax and benefit provisions will be maintained in their current form. The BKR estimate is approximately $23 \%$ larger than the number calculated via the SSA's wage-growth valuation formula, which values wage growth based on an assumed, time-invariant, growth rate of the economy-wide average wage.

To be clear, the sole difference between the BKR and Social Security OAI valuations in the BKR study, as opposed to the current study, is the method used to value the wage growth security. I.e., BKR employ a micro-based strategy in determining and valuing the idiosyncratic component of the worker's primary insurance amount, which is similar to the method that Social Security would use to understand expected differences across workers in their future Social Security benefits and taxes. And to focus attention solely on the method of valuing the wage-growth security, BKR, in forming both their own and the Social Security valuations of the total unfunded operating liability to Americans of working age in 2005, BKR value/measure each worker's idiosyncratic component the same way. As BKR discuss, the main reason that the two valuations differ is that Social Security discounts future average wage growth at its assumed 2.9 percent safe rate, whereas BKR use the prevailing, in 2005, term structure of yields on Treasury Inflation Protected Securities (TIPS). The TIPS yields in 2005 varied from around $1 \%$, at the short-end of the term structure, to $2.4 \%$ at the long, i.e., 30 -year end of the term structure.

The valuation differences depend, then, in large part on how Social Security values safety 
as opposed to risk. Take a worker who is age 58 and is expected to start collecting benefits at age 62. Although his ultimate PIA remains uncertain, once his received benefit is received, it will be paid for sure as long as he lives. I.e., it will be paid in the form of an inflation-protected annuity. This is an annuity being provided by the government and the only appropriate discount rate(s) to use to value such an annuity is the prevailing TIPS term structure. This is an important difference not only between how Social Security forms its valuations, but also, as we'll indicate momentarily, how GZ formulates their valuation. GZ follows Social Security in assuming a fixed 2.9 percent real rate of discount.

In the current paper, we too adopt the 2.9 percent real discount rate, but the reason for doing so is that the vast majority of, in this case, OASDI future benefits and taxes lie in the very distant future. Since the longest maturity TIPS is now less than 30 years and since the TIPS yields rise sharply with maturity, it's quite possible that were, say, 50-year TIPS to be floated on the market, they would yield $2.9 \%$ after inflation. Because of this uncertainty and to help limit the possible sources of differences between our open group unfunded liability estimate and SSA's, we use the 2.9 percent rate here, while maintaining that doing so in the BKR study, which is looking at shorter-term liabilities would have been inappropriate.

In contrast to the BKR conclusion Social Security is understating its liabilities, GZ suggest the opposite is true. GZ estimate the OAI accrued benefit liability to be $20 \%$ less than would arise based on SSA's valuation methodology. As indicated, GZ assume a $2.9 \%$ safe real discount rate notwithstanding the fact that they are considering relatively short-term liabilities. Simply using the prevailing TIPS term structure could, potentially, overturn their main finding. This is not to suggest that using the TIPS term structure of yields is precisely the right way to discount real cash flows provided by the government. The reported yields are calculated under the assumption that the coupons on the TIPS can be reinvested at the same internal rate of return. What's really needed to precisely value safe (as in government provided) real cash flows of different maturities is the prevailing prices on zero-coupon TIPS. Unfortunately, there is no 
zero-coupon TIPS market from which to cull these prices.

The GZ approach to valuing wage growth also differs from that in BKR on several fronts. Whereas BKR assume a simple linear relationship between aggregate wage growth rates and asset returns, GZ model the log wage-growth rate over a horizon of $h$ periods as a function of the current log wage-dividend ratio:

$$
w_{t+h}-w_{t}=\left(g_{w}-\frac{\sigma_{w}^{2}}{2}\right) h-\kappa\left(w_{t}-d_{t}-\bar{w} d\right) h+\sigma_{w} \epsilon_{w, t}
$$

where $g_{w}$ and $\sigma_{w}$ are the growth rate and volatility of the wage process, respectively, $d_{t}$ is the the value of dividends at time $t$ and $\bar{w} d$ is the long-run wage-dividend ratio. GZ use this meanreverting specification to capture the long-run relationship between stock markets and wages. However, upon closer inspection, one can see that (4) appears to suggest that the log-wage growth rate is a function of the relative levels of wages and dividends. This is not the same as (2), which is a regression strictly in rates. To see that (4) is focused on predicting the level of future wages, not that it can be rewritten as an $h$ th-order autoregressive distributed lag (ADL) with a deterministic time trend:

$$
w_{t+h}=\left(g_{w}-\frac{\sigma_{w}^{2}}{2}+\kappa \bar{w} d\right) h+(1-\kappa) w_{t}+\kappa d_{t}+\sigma_{w} \epsilon_{w, t}
$$

Note that (5) places very stringent restrictions on the manner in which the current value of the stock market, which GZ assume is proportional to dividends, relates to the level of future wages. There also seems to be some confusion in the GZ paper about whether the study is focused on the future level of aggregate wages or wages per worker. Similarly, there is a flipping back and forth between the level of total dividends and the dividend-price ratio. These concerns aside, GK and BKR do firmly agree on their key point, namely that government obligations to and claims on the public are risky and need to be valued taking that risk into account. 
Since Social Security is an ongoing enterprise, the most important liability to be measured is not what's owed just to current workers or what would be owed were the system to be closed down, at the margin, but what the system owns, in total and on net, in continuing to operate in its current form. This is the lability to whose measurement we now turn.

\section{Methodology}

As previously mentioned, the techniques employed in this paper are closely related to those in BKR. The major differences is that we are focusing on OASDI, not just OAI, i.e., we are focusing on the entire program and we are calculating the open group net liability by working with aggregate total outflows and inflows of the system over the infinite horizon. So we are considering growth rate securities where the growth rate is not with respect to a per capita value, like the economy-wide average wage, but rather with respect to aggregate amounts of benefits and tax payments. Thus there are no demographic cohorts or idiosyncratic components that need to be weighted and summed to form the aggregate liability. Formally, the open group net liability at time $t$, denoted $\ell_{t}$, is defined as follows:

$$
\ell_{t}=P V_{t}(\text { Future Outflows })-P V_{t}(\text { Future Inflows }),
$$

where $P V_{t}(\cdot)$ is the risk-adjusted present value operator given information up to time $t$. Let $y_{T}$ denote the outflow at time $T$. Note that this future quantity is related to the current value, $y_{t}$, via the ensuring growth rates as follows:

$$
y_{T}=y_{t} \prod_{i=1}^{T}\left(1+g_{y, t+i}\right) \text {, }
$$

where $g_{y, t+i)}$ is the growth rate of aggregate benefit payments between periods $t$ and $t+1$. Establishing an analogous relationship for the total inflow, referenced by $z$, the equation for $\ell$ 
becomes:

$$
\begin{aligned}
\ell_{t} & =P V_{t}\left(\sum_{i=1}^{\infty} y_{t} \prod_{j=1}^{i}\left(1+g_{y, t+j}\right)\right)-P V_{t}\left(\sum_{i=1}^{\infty} z_{t} \prod_{j=1}^{i}\left(1+g_{z, t+j}\right)\right) \\
& =y_{t} \sum_{i=1}^{\infty} P V_{t}\left(\prod_{j=1}^{i}\left(1+g_{y, t+j}\right)\right)-z_{t} \sum_{i=1}^{\infty} P V_{t}\left(\prod_{j=1}^{i}\left(1+g_{z, t+j}\right)\right)
\end{aligned}
$$

where the second equality follows from the fact that $y_{t}$ and $z_{t}$ are known at time $t$ and that the $P V(\cdot)$ function is a linear operator.

As equation (7) shows, the key to calculating the open group unfunded liability lies in calculating the present value of the product of future growth-rate factors, which are unknown in the current period. This is where the BKR method has an intuitive appeal. Suppose there exists a security that pays a return equal to the growth rate of the total outflow from the social security system over the next period. That is, a one dollar investment in this security at time $t$ will pay the holder $1+g_{y, t+1}$ at time $t+1$. A two-period security will pay the holder $\left(1+g_{y, t+1}\right)\left(1+g_{y, t+2}\right)$ at time $t+2$. Continuing in this fashion, one can see that $\ell_{t}$ is just the sum of the prices of these securities. Let $G_{y, t, i}$ denote the value of this hypothetical security, where $y$ indicates the growth rate on which the payoff is based, $t$ indicates the current time, and $i$ indicates the number of periods to maturity. Then the unfunded obligation formula can be rewritten as follows:

$$
\ell_{t}=y_{t} \sum_{i=1}^{\infty} G_{y, t, i}-z_{t} \sum_{i=1}^{\infty} G_{z, t, i}
$$

Note that $\ell_{t}$ is well-defined so long as the infinite sums converge, which means that the price of the growth rate security must converge to zero as the number of holding periods increases to infinity.

Equation (8) indicates that all we need in order to calculate the unfunded liability are the values of the growth rate securities. First consider the value of the one period security. If the price of this asset was determined in financial markets, we know from the First Fundamental 
Theorem of Asset Pricing that $G_{y, t, 1}$ equals the expected payout at time $t+1$, discounted at the risk-free rate of return, under the equivalent martingale probability measure ${ }^{3}$ :

$$
G_{y, t, 1}=\mathrm{E}_{t}^{\mathcal{Q}}\left[\frac{1+g_{y, t+1}}{1+r}\right]
$$

Now suppose we regress the outflow or inflow one-period growth rate on a constant and the contemporaneous returns from $n$ traded securities as in (2). Assuming that this regression captures all of the aspects of the outflow growth rate that are priced by the market, except for an idiosyncratic noise term that is uncorrelated with the asset returns, then we can move (2) one period forward, add one to both sides, and then take expectations under the risk-neutral measure:

$$
\begin{aligned}
\mathrm{E}_{t}^{\mathcal{Q}}\left[1+g_{y, t+1}\right] & =\mathrm{E}_{t}^{\mathcal{Q}}\left[1+\alpha+\sum_{i=1}^{n} \beta_{i} s_{i, t+1}+\epsilon_{t+1}\right] \\
& =1+\alpha+r \sum_{i=1}^{n} \beta_{i}
\end{aligned}
$$

where the second equality follows from the fact that the expected return on any traded asset under the risk-neutral measure equals the risk-free rate. Plugging this result into (9), we see that

$$
\begin{aligned}
G_{y, t, 1} & =\frac{1}{1+r} \mathrm{E}_{t}^{\mathcal{Q}}\left[1+g_{y, t+1}\right] \\
& =\frac{1+\alpha+r \sum_{i=1}^{n} \beta_{i}}{1+r}
\end{aligned}
$$

This is the result derived in BKR. Note that the right hand side of (11) is independent of time, which suggests that the price of the wage security with one period to maturity is the same regardless of the current time. That is, $G_{y, t, 1}=G_{y, u, 1}=G_{y, 1}$ for any times $t$ and $u$.

\footnotetext{
${ }^{3}$ Throughout the paper, the terms "equivalent martingale measure," the "risk-neutral" measure, and the "Q " measure are used interchangably.
} 
Now consider the same security but with two periods remaining to maturity. The payoff in two periods equals $\left(1+g_{y, t+1}\right)\left(1+g_{y, t+2}\right)$, implying that its price equals

$$
\begin{aligned}
G_{y, t, 2} & =\mathrm{E}_{t}^{\mathcal{Q}}\left[\frac{\left(1+g_{y, t+1}\right)\left(1+g_{y, t+2}\right)}{(1+r)^{2}}\right] \\
& =\mathrm{E}_{t}^{\mathcal{Q}}\left[\frac{1+g_{y, t+1}}{1+r} \mathrm{E}_{t+1}^{\mathcal{Q}}\left[\frac{1+g_{y, t+2}}{1+r}\right]\right] \\
& =\mathrm{E}_{t}^{\mathcal{Q}}\left[\frac{1+g_{y, t+1}}{1+r} G_{y, t+1,1}\right] \\
& =G_{y, 1} \mathrm{E}_{t}^{\mathcal{Q}}\left[\frac{1+g_{y, t+1}}{1+r}\right] \\
& =G_{y, 1}^{2}
\end{aligned}
$$

where the second equality follows from the law of iterated expectations. Notice that $G_{y, t, 2}$ is also independent of time. As shown in the appendix, the two-period case extends analogously to the $T$ period case so that

$$
G_{y, T}=\mathrm{E}_{t}^{\mathcal{Q}}\left[\prod_{j=1}^{T}\left(\frac{1+g_{y, t+j}}{1+r}\right)\right]=G_{y, 1}^{T}=\left(\frac{1+\alpha+r \sum_{i=1}^{n} \beta_{i}}{1+r}\right)^{T}
$$

This pricing formula satisfies the convergence requirements of (8) so long as the following relationship between $r$ and the regression parameters holds

$$
\frac{1+\alpha+r \sum_{i=1}^{n} \beta_{i}}{1+r} \leq 1 \Longleftrightarrow \frac{\alpha}{1-\sum_{i=1}^{n} \beta_{i}} \leq r
$$

Equation (13) provides a means of generating an estimate of the open group net liability using only contemporaneous asset returns. However, as GZ have argued in the case of wages, the growth rates of 1 social security outflows and inflows may have little correlation with current real asset returns. This conjecture is supported by our contemporaneous regressions, not shown, for social security benefits and contributions where the parameters on the returns are not significantly different from zero and the $R^{2}$ values are very small. However, the correlation over 
longer horizons is likely to be significant. This is the relationship that GZ try to capture with their mean-reverting structure. As (5) shows, however, their efforts can simply be reformulated as an autoregressive distributed lag model in wage levels. We suggest keeping the regression in growth rates for simplicity and flexibility. One approach, taken by BKR, is to extend (??) to incorporate lagged asset returns. That is, one can regress outflow growth rates on a constant and asset returns from $h$ periods ago:

$$
g_{y, t}=\alpha+\sum_{i=1}^{n} \beta_{i} s_{i, t-h}+\epsilon_{t}
$$

The general $T$ period adjusted growth rate security valuation, derived in the appendix, is given by

$$
G_{y, t, T}=\left(\frac{1+\alpha+r \sum_{i=1}^{n} \beta_{i}}{1+r}\right)^{\max (T-h, 0)} \cdot \prod_{j=1}^{\min (h, T)}\left(\frac{1+\alpha+\sum_{i=1}^{n} \beta_{i} s_{i, t+j-h}}{1+r}\right)
$$

This asset price converges to zero with the length of maturity of the asset under the same conditions as (13). Note that (14) does not allow for both contemporaneous and lagged values of asset returns on the right-hand-side. Moreover, it does not allow more than one lag length to explain outflow or inflow growth rates. These restrictions allow the growth rate security to be priced without making strong assumptions about the covariance structure of higher order moments of asset returns. Indeed, if one doesn't limit the regression to include only assets returns that arise in one and only one period (be that period the current period of a period in the past), one ends up with asset pricing formulas that are remarkably complex and exceptionally demanding in terms of estimation. So using lagged regressors is important, based on what the data tell us, but we're stuck with the theory effectively restricting us to trying one lag length at a time. Thus, while we use the BKR approach below, we also consider two other approaches to the pricing of the growth rate securities.

Before explaining these two approaches, let us belabor the problem we face here via an example. Suppose we are trying to price a 1-quarter aggregate benefits growth-rate security, i.e., 
a security that pays, in one quarter, 1 times the growth-rate factor over the next quarter. If we estimate a regression of guarterly aggregate benefit growth rates against, say, security returns lagged five periods, there is no problem in deriving a simple pricing formula for the security. Nor is there a problem if we include a number of lags in the regression. The problem arises if we include more than one lag in this regression and try to use the results from this 1-quarter growthrate regression to price assets of other maturities, say seven quarters. Our proposed solution, for which we have two variants (KK1 and KK2), then, involves running separate regressions for each maturity. A simple way to think of this is to view growth-rate securities of one maturity as completely different from growth-rate securities of a different maturity. Thus we can call a 15 quarter aggregate benefits growth rate security the apple growth rate security and a 22-quarter aggregate benefits growth rate security as the banana growth rate security. If the growth rates in question were really about growth in apple consumption over 15 quarters and growth in banana consumption over 22 quarters, and there were no apple growth rate securities of any other maturity nor any banana growth-rate security of any other maturity, we'd simply price the apples growth-rate security on its own and the bananas growth-rate security on its own. In this case, the problem we are raising would never come up because we wouldn't immediately connect the growth rate in apples of 15 quarters with the growth rate in bananas over 22 quarters.

Returning to the specifics, our two KK1 and KK2 approaches are based on the observation that we can view an $h$ period growth-rate security as in equation (13) as delivering the product of $h$ period-specific growth-rate factors or we can view it delivering a single $h$ period growth rate. That is,

$$
G_{y, t, h}=\mathrm{E}_{t}^{\mathcal{Q}}\left[\prod_{j=1}^{h}\left(\frac{1+g_{y, t+j}}{1+r}\right)\right] \equiv \mathrm{E}_{t}^{\mathcal{Q}}\left[\frac{1+g_{y, h, t+h}}{(1+r)^{h}}\right]
$$

This equivalence implies that instead of modeling the one quarter growth rates we can model the growth rates over more general time horizons. In theory, the absence of arbitrage implies that these two expectations have the same value. That is, if the true parameters of either regression 
are known, then the security prices of both methods will be identical. In practice, however, regressions are inevitably estimated with finite data sets, which can cause differences in these values. Intuitively, the regression that produces more accurate parameters will also produce a more accurate value of the growth rate security.

To model growth rates over longer horizons, we employ two variations of the BKR technique. In the first method, which we the call the KK1 method, we regress growth rates over a general time interval against the one period asset returns over the same horizon. That is, for an $h$-period horizon, we regress

$$
g_{y, h, t}=\alpha_{h}+\sum_{i=1}^{n} \sum_{j=0}^{h-1} \beta_{h, i j} s_{i, t-j}+\epsilon_{t}
$$

where $g_{y, h, t}$ is the growth rate of $y$ from time $t-h$ to $t$. Note that for $h=1,(17)$ is identical to (??). Using this regression, we can calculate the value of the corresponding $h$-period growth rate security. Specifically,

$$
\begin{aligned}
G_{y, t, h} & =\mathrm{E}_{t}^{\mathcal{Q}}\left[\frac{1+g_{y, h, t+h}}{(1+r)^{h}}\right] \\
& =\mathrm{E}_{t}^{\mathcal{Q}}\left[\frac{1+\alpha_{h}+\sum_{i=1}^{n} \sum_{j=0}^{h-1} \beta_{h, i j} s_{i, t+h-j}+\epsilon_{t+h}}{(1+r)^{h}}\right] \\
& =\frac{1+\alpha_{h}+r \sum_{i=1}^{n} \sum_{j=0}^{h-1} \beta_{h, i j}}{(1+r)^{h}}
\end{aligned}
$$

Notice that $G_{y, t, h}$ depends on the parameters from the $h$-period regression. Rather than using one set of regression parameters to value the growth rate security for any number of holding periods, as in (13), we run a separate regression for each investment horizon. That is, the $T$-period security is valued using parameters from (17) with $h=T$. This contrasts with the $T+1$-period security which utilizes the estimated coefficients from a separate regression (17) with $h=T+1$.

To use the KK1 method to calculate $\ell_{t}$ using (8), one would theoretically have to run 
regressions for $h=1,2, \ldots, \infty$ in order to calculate all of the growth rate security prices. However, the finiteness of the data set requires that we make an appropriate approximation. To this end, we calculate the first $n$ growth security prices and then fit a line of exponential decay for the remaining prices ${ }^{4}$. So long as the estimated prices exhibit negative curvature, the approximated infinite sums will converge to zero and (8) is well defined. According to equation (18), the growth security prices will converge to zero as the holding period increases to infinity provided that $\alpha_{h}$ and $\sum_{i=1}^{n} \sum_{j=0}^{h-1} \beta_{h, i j}$ converge to a constant. Even if they explode, they need only approach infinity at a rate slower than $(1+r)^{h}$.

A potential drawback of the regressions in (17) is that to calculate the value of an $h$ holding period growth rate security, the number of regressors on the right hand side is equal to $n \times h+1$. For example, with just two assets there will be 31 parameters to be estimated to value the 15 period growth rate security. Depending on the length of the time series, one might have difficulty estimating these parameters with any meaningful precision. As an alternative to regressing $h$ period growth rates on $h$ one period growth rates, we also regress $h$ period growth rates on $h$ period returns. We call this the KK2 method. Specifically, (17) becomes

$$
g_{y, h, t}=\alpha_{h}+\sum_{i=1}^{n} \beta_{h, i} s_{i, h, t}+\epsilon_{t}
$$

For example, if $h=8$, we regress the eight period growth rate on the eight period asset returns. Equation (19) implies that the number of regressors is always equal to $n+1$, which is independent of the number of holding periods. The growth rate security formula is

$$
\begin{aligned}
G_{y, t, h} & =\mathrm{E}_{t}^{\mathcal{Q}}\left[\frac{1+g_{y, h, t+h}}{(1+r)^{h}}\right] \\
& =\mathrm{E}_{t}^{\mathcal{Q}}\left[\frac{1+\alpha_{h}+\sum_{i=1}^{n} \beta_{h, i} s_{i, h, t+h}+\epsilon_{t+h}}{(1+r)^{h}}\right] \\
& =\frac{1+\alpha_{h}+\left[(1+r)^{h}-1\right] \sum_{i=1}^{n} \beta_{h, i}}{(1+r)^{h}},
\end{aligned}
$$

\footnotetext{
${ }^{4}$ Specifically, we fit a line of the form $y_{t}=a e^{b t}$ where the parameters $a$ and $b$ are determined via standard non-linear least squares techniques.
} 
where $(1+r)^{h}-1$ is the $h$ period risk-free return.

An important implication of (20) is that as the holding period increases, the price only converges to zero if the sum of the betas converges to zero. To see that this is the case, consider the regression for just one asset return

$$
g_{y, h, t}=\alpha_{h}+\beta_{h} s_{h, t}+\epsilon_{t}
$$

The OLS estimate for $\beta_{h}$ is

$$
\hat{\beta}_{h}=\frac{\frac{1}{T} \sum_{t=1}^{T}\left(s_{h, t}-\bar{s}_{h}\right)\left(g_{y, h, t}-\bar{g}_{y, h}\right)}{\frac{1}{T} \sum_{t=1}^{T}\left(s_{h, t}-\bar{s}_{h}\right)^{2}}
$$

Note that the denominator of $\hat{\beta}_{h}$ is the sample variance of the asset return. Assuming that the asset's returns over any time interval are non-stationary with a variance that strictly increases with the length of the interval, then the variance approaches infinity as the return horizon increases. This implies that $\hat{\beta}_{h}$ converges to zero as $h$ increases. This same argument applies for a regression with multiple assets. Therefore, the growth rate security prices produced by (20) do converge to zero, which implies that the equation for $\ell_{t}$ is well defined.

The major appeal of the KK1 and KK2 approaches is that, as in the BKR paper, the ultimate quantity of interest, namely the growth rate security price, is only dependent on $\alpha$, the $\beta_{i}$, and the risk-free rate $r$. That is, the valuation formulas for $\ell_{t}$ are completely independent of any mean reversion, volatility, or other parameters related to either the outflow and inflow processes or the asset returns processes. Since the regressions provide estimates of $\alpha$ and the $\beta_{i}$, the only remaining variable to be determined is $r$. One of the main objections to the BKR results, raised by Geanakoplos and Zeldes, is that they seem to be driven in large part by the risk-free rate term structure employed. Specifically, as indicated above, BKR used the Treasury Inflation Protected Securities (TIPS) term structure at various horizons to discount the relevant 
cash flows. This contrasts with the constant $2.9 \%$ rate used by both the SSA and GZ. The problem with using the TIPS term structure, as previously mentioned, is that it is composed of yields to maturity which, by definition, depend on the periodic coupon payments that occur over the life of the security in addition to the principle payment made at maturity. Therefore, these yields represent a weighted average of spot rates and, consequently, with an rising term structure of yields, the yield to maturity for a bond of maturity $t$ will always underestimate the actual spot rate at time $t .^{5}$. As a result, the BKR appear to be discounting safe streams at rates that are somewhat lower than is appropriate. Since zero-coupon TIPS prices are not available for different maturities and since we are valuing streams into the distant future, we choose in this paper to use the constant risk-free rate of $2.9 \%$. This choice will ensure that our estimate of SSA's open group liability does not reflect differences in assume safe interest rates. Note that in the KK1 and KK2 techniques, the ideal risk-free rate would reflect term to maturity of the growth rate securities being calculated. By using a constant $2.9 \%$ for all maturities, we implicitly assume a flat spot rate curve which will likely under-estimate the values of the growth rate securities at short horizons and, therefore, under-estimate the open group net liability.

\section{Results}

To put the methodology described in the previous section to use, we employ data provided by the SSA's website ${ }^{6}$. For the outflow variable, $y$, and inflow variable, $z$, we use quarterly benefit payments and net contributions, respectively, from the first quarter of 1987 until the first quarter of 2009 for a total of 89 observations. These data were then converted to real dollars for our base period, the first quarter of 2009, using the "All Urban Consumers" Consumer Price Index available from the Bureau of Labor Statistics website ${ }^{7}$. The real values were then

\footnotetext{
${ }^{5}$ A spot rate for a given maturity, often called a "zero" rate, is the rate of interest earned on a bond which pays only a single payment at maturity with no intermediate cash flows (i.e. the rate on a zero-coupon bond).

${ }^{6}$ The data was downloaded from http://www.ssa.gov/OACT/ProgData/tsOps.html.

${ }^{7} \mathrm{http}: / /$ www.bls.gov/cpi/
} 
seasonally adjusted via the X-12 ARIMA filter used and provided by the Census Bureau ${ }^{8}$. For the asset returns data, we used quarterly returns for the S\&P 500 Indexes and the Merrill Lynch Three Month Treasury and 10 Year Plus total return indexes. All of this data was obtained from a Datastream terminal. The nominal returns were converted to real returns using the same CPI as the social security data.

An important implication of the KK1 and KK2 regressions, shown in equations (17) and (19), respectively, is that constructing a time series of non-overlaping growth rates requires many data points. Suppose the original time series, i.e. the raw data before calculating growth rates, has $\mathrm{N}$ observations. Using this series to construct growth rates in the dependent variable produces $\frac{N}{h+1}$ usable observations. This implies that the data set is severely reduced even for small values of $h$. To alleviate this problem, we use overlapping windows to construct the growth-rate series. For example, if the first data point in our series is 1990-Q1, then the first growth rate observation for $h=4$ quarters is the growth rate from 1990-Q1 to 1991-Q1. The second observation is the growth rate from 1990-Q2 to 1991-Q2, rather than 1991-Q1 to 1992-Q2. Continuing this way, the total number of growth rate observations is $N-h$, which decreases with $h$ much slower than $\frac{N}{h+1}$. We also construct the multi-period horizon returns for the KK2 regressions in this manner. Since we will be using ordinary least squares (OLS) to estimate the parameters of the model, it is important to point out that constructing the data in this way imposes a non i.i.d. relationship among the error terms. However, the purpose of the regressions is not to test hypotheses regarding any of the parameters, but rather to use the resulting estimates as a measure of the relationship between social security cash flows and financial returns. Even in the presence of heteroskedasticity and serially correlated errors, the OLS estimates remain consistent.

The results are presented in Tables 1 through 3 . We only present the estimates of the net liability because the regressions themselves are merely a means to an end. All dollar amounts

\footnotetext{
${ }^{8}$ http://www.census.gov/srd/www/x12a/
} 
are trillions of 2009-Q1 dollars. For each of the three methods described in the previous section, we use three different combinations of regressors in addition to a constant:

(I) S\&P 500 Index

(II) S\&P 500 and Merrill Lynch Three Month T-Bill Total Returns Indexes

(III) S\&P 500 Index and the Merrill Lynch Three Month T-Bill and 10 Year Plus Bond Total Returns Indexes

The columns of Tables 1 through 3 are headed by these roman numerals indicating to which set of regressors the results belong.

The regression results for the BKR-style regressions, i.e. equations (??) and (14), are presented in Table 1. Both panels show the present value of benefits paid and net contributions received as well as the net uncovered liability. In Panel A, the estimates are based on the regression whose lagged returns produce the highest possible value of $R^{2}$, which is provided in parenthesis underneath the present value estimates ${ }^{9}$. That is, we run regression (14) for $h=0,1, \ldots, 40$ and plug the parameters from the regression with the highest $R^{2}$ into (15). For regression (I), the net liability is positive and equal to $\$ 62.439$ trillion. This contrasts with the estimates from regressions (II) and (III) which indicate a net surplus, meaning that the present value of future contributions exceeds that of future benefits.

Panel B of Table 1 presents the estimates using the average across all lag lengths. This is a useful measure for the BKR-style regressions because there is considerable variance in the estimated values of the benefits and contributions depending on which lag is used. Some lags produce deficits while others produce surpluses. However, as Panel B shows, the average values for all regressors indicate a deficit in the range of $\$ 74$ to $\$ 84$ trillion ${ }^{10}$. The standard errors for

\footnotetext{
${ }^{9}$ The value of the adjusted $R^{2}$, often denoted $\bar{R}^{2}$, closely tracks that of $R^{2}$ and, accordingly, the results are the same using either the highest $R^{2}$ or $\bar{R}^{2}$.

${ }^{10}$ Note that the averages exclude any cases where the parameters do not meet the convergence requirement previously discussed. This only occurred in regressions (II) and (III) and for only a couple values of $h$. As a result of these exclusions, the net obligation in Panel B need not be the difference between the benefits and contributions.
} 
Table 1: BKR-Style Regression Results

Panel A: Values Chosen at Lag with Highest $R^{2}$

\begin{tabular}{|l|rrr|}
\hline & \multicolumn{1}{|c}{$(\mathrm{I})$} & \multicolumn{1}{c|}{$(\mathrm{II})$} & \multicolumn{1}{c|}{$(\mathrm{III})$} \\
\hline Benefits & $\$ 128.193$ & $\$ 81.986$ & $\$ 152.602$ \\
& $(0.064)$ & $(0.089)$ & $(0.096)$ \\
Contributions & $\$ 65.753$ & $\$ 139.068$ & $\$ 286.180$ \\
& $(0.074)$ & $(0.085)$ & $(0.209)$ \\
\hline Net Obligation & $\mathbf{\$ 6 2 . 4 3 9}$ & $\mathbf{- \$ 5 7 . 0 8 2}$ & $\mathbf{- \$ 1 3 3 . 5 7 8}$ \\
\hline
\end{tabular}

Panel B: Average Values Across all Lags

\begin{tabular}{|l|rrr|}
\hline Benefits & $\$ 204.573$ & $\$ 206.654$ & $\$ 189.371$ \\
& $(\$ 9.159)$ & $(\$ 27.953)$ & $(\$ 21.928)$ \\
Contributions & $\$ 125.247$ & $\$ 140.234$ & $\$ 93.177$ \\
& $(\$ 10.620)$ & $(\$ 23.756)$ & $(\$ 9.368)$ \\
\hline Net Obligation & $\mathbf{\$ 7 9 . 3 2 6}$ & $\mathbf{\$ 7 4 . 8 6 0}$ & $\mathbf{\$ 8 3 . 3 4 5}$ \\
\hline
\end{tabular}

the averages are presented in parenthesis ${ }^{11}$.

Table 2 contains the results for the second method. Recall that for this approach, the price of the $h$-quarter growth rate security is calculated with parameters from a regression of $h$-quarter growth rates on each of the one quarter returns during that period. After calculating the first $n$ growth rate securities, a line of exponential decay can be fitted to approximate the remaining terms in the sum that cannot be calculated via regression. Table 2 shows the results for $n=15,20$, and 25 . The net liability estimates when the S\&P 500 is the only regressor are all positive and are reasonably close together in the range of $\$ 65$ to $\$ 76$ trillion. However, as with the BKR method, adding the bond indexes to the regressions makes the results unstable. The regression (II) results nearly triple in going from $n=15$ to $n=20$. Moreover, the benefits diverge for $n=25$ leading to an exploding deficit. The estimates based on (III) are even more unstable except in the opposite direction. In that case the contributions diverge even for $n=15$. Note that it was not possible to run regressions for holding periods beyond 20 quarters in case (III) because the number of regressors relative to the sample size caused errors in the matrix inversion necessary for ordinary least squares.

\footnotetext{
${ }^{11}$ These are not the standard errors of the parameters, but are just simple standard errors for the sample average dollar estimate.
} 
Table 2: Long Growth Rates on Short Returns (KK1 Method)

\begin{tabular}{|c|c|c|c|c|}
\hline & & (I) & (II) & (III) \\
\hline \multirow[t]{3}{*}{$n=15$} & Benefits & $\$ 133.358$ & $\$ 144.966$ & $\$ 55.697$ \\
\hline & Contributions & $\$ 57.987$ & $\$ 90.691$ & Exploding \\
\hline & Net Obligation & $\$ 75.372$ & $\$ 49.645$ & Exploding Surplus \\
\hline \multirow[t]{3}{*}{$n=20$} & Benefits & $\$ 130.929$ & $\$ 240.144$ & $\$ 42.126$ \\
\hline & Contributions & $\$ 57.474$ & $\$ 85.397$ & Exploding \\
\hline & Net Obligation & $\$ 73.456$ & $\$ 147.076$ & Exploding Surplus \\
\hline \multirow[t]{3}{*}{$n=25$} & Benefits & $\$ 124.065$ & Exploding & - \\
\hline & Contributions & $\$ 58.476$ & $\$ 78.361$ & - \\
\hline & Net Obligation & $\$ 65.589$ & Exploding Deficit & - \\
\hline
\end{tabular}

The results for the third approach are presented in Table 3 . Here the number of regressors does not grow with the holding period of the growth rate security. As a result, one can calculate the values of these securities beyond $n=25$ quarters. Table 3 shows results for $n=15,20,25$, 30, 35, and 40 quarters. For regression (I) with $n=15,20$, and 25, and for regression (II) with $n=15$ this method produces net liability estimates that are very similar to those in Table 2 . However, beyond $n=25$ the benefits and contributions decrease and increase, respectively, in a uniform fashion which leads to a decreasing and ultimately negative net liability. Moreover, regression (III) appears to be unstable and the contributions diverge for all values of $n$.

As Tables 1 through 3 show, the results are inconclusive. For regression (I), which contains the $\mathrm{S} \& \mathrm{P} 500$ as the only stochastic regressor, the net liability is positive and consistently between $\$ 58.4$ and $\$ 79.3$ trillion across all tables except in Table 3 for $n$ greater than 25 . Compared with $\$ 15.1$ trillion, these estimates imply that the SSA underestimates its unfunded liability by as much as $\$ 41.3$ to $\$ 64.2$ trillion. For regressions (II) the net obligation is considerably more variable and ranges from positive infinity to negative infinity. The finite estimates for regression (II) range from $\$ 49.6$ to $\$ 147.076$ trillion on the positive side and $-\$ 57.082$ to $-\$ 404.075$ trillion on the negative side. Lastly, the third set of regressors produces even more unreliable results. The KK1 and KK2 methods imply exploding surpluses across all values of $n$.

There are several reasons why the KK1 and KK2 methods produce such highly variable 
Table 3: Long Growth Rates on Long Returns (KK2 Method)

\begin{tabular}{|c|c|c|c|c|}
\hline & & (I) & (II) & (III) \\
\hline \multirow[t]{3}{*}{$n=15$} & Benefits & $\$ 131.161$ & $\$ 143.848$ & $\$ 84.062$ \\
\hline & Contributions & $\$ 61.361$ & $\$ 97.144$ & Exploding \\
\hline & Net Obligation & $\$ 69.800$ & $\$ 46.704$ & Exploding Surplus \\
\hline \multirow{3}{*}{$n=20$} & Benefits & $\$ 129.720$ & $\$ 147.546$ & $\$ 80.276$ \\
\hline & Contributions & $\$ 60.964$ & $\$ 122.045$ & Exploding \\
\hline & Net Obligation & $\$ 68.757$ & $\$ 25.501$ & Exploding Surplus \\
\hline \multirow[t]{3}{*}{$n=25$} & Benefits & $\$ 121.542$ & $\$ 133.485$ & $\$ 76.151$ \\
\hline & Contributions & $\$ 63.141$ & $\$ 197.610$ & Exploding \\
\hline & Net Obligation & $\$ 58.401$ & $-\$ 64.124$ & Exploding Surplus \\
\hline \multirow[t]{3}{*}{$n=30$} & Benefits & $\$ 112.095$ & $\$ 110.794$ & $\$ 65.109$ \\
\hline & Contributions & $\$ 69.953$ & $\$ 329.877$ & Exploding \\
\hline & Net Obligation & $\$ 42.142$ & $-\$ 219.083$ & Exploding Surplus \\
\hline \multirow[t]{3}{*}{$n=35$} & Benefits & $\$ 103.821$ & $\$ 96.291$ & $\$ 63.330$ \\
\hline & Contributions & $\$ 81.011$ & $\$ 500.366$ & Exploding \\
\hline & Net Obligation & $\$ 22.809$ & $-\$ 404.075$ & Exploding Surplus \\
\hline \multirow[t]{3}{*}{$n=40$} & Benefits & $\$ 95.442$ & $\$ 88.820$ & $\$ 64.719$ \\
\hline & Contributions & $\$ 101.968$ & Exploding & Exploding \\
\hline & Net Obligation & $-\$ 6.526$ & Exploding Surplus & Exploding Surplus \\
\hline
\end{tabular}

estimates of the unfunded obligation. First and foremost, the original data set has only 89 points.

This coupled with the reuse of data via overlapping horizons may introduce considerable small sample bias into the OLS parameter estimates, especially when valuing a growth rate security with a long holding period. Moreover, the process of fitting an exponential decay for the first $n$ maturities of the growth rate security may be too coarse an approximation. Finite sample noise may cause price estimates to fluctuate up or down as $h$ increases. Therefore, the first $n$ prices may not accurately reflect true exponential decay or even be able to establish the existence of such a relationship.

Another potentially major factor influencing our results is the coming demographic shift in the US population. In abstracting away from all the cohort growth rates that the SSA uses, our methods put the burden of capturing this very important change on financial markets. That is, we rely on any swells or dips in asset returns to at least partially coincide with major shifts in the composition of the population. There are many papers that provide some support for this assumption. Bakshi and Chen (1994), for example, find that an increase in the average 
age of a population predicts an increase in the premium for risky assets. Further support for a substantial relationship between asset prices and demographics is provided in Bergantino (1998). On the other hand, Poterba (2001) argues that it is difficult to find such a robust relationship. Therefore, it is difficult to characterize the extent to which our assumption is accurate or not. The only certainty is that the demographic shift will occur as the baby boomers retire and that this will affect future social security benefit and contribution cash flows. If the statistical relationship between demographics and financial markets is weak, as suggested by Poterba, then our open group unfunded obligation estimates could be biased either upwards or downwards.

\section{Conclusions}

In this paper, we estimate the value of the US SSA's open group unfunded old age, survivors, and disability insurance obligation. This quantity equals the difference between the present value of all future benefit payments and contribution receipts. We believe that this metric is the most appropriate means by which one can assess the overall health of the social security system. Unfortunately, given the infinite horizon over which the estimate is formed, it is the most difficult metric to measure. The SSA takes an actuarial approach and makes three sets of assumptions regarding various demographic growth rates. They then project current numbers forward through time using these constant rate assumptions and then discount the benefit and contribution cash flows back to the present using a constant interest rate. Their latest official estimate of the unfunded liability is $\$ 15.1$ trillion.

We believe that the actuarial approach, while functional, is too crude because it does not treat benefits and contributions as risky cash flows. While the Trustees Report does supplement its actuarial estimates with simulations, their stochastic modeling is based on traditional time series methods. That is, variables are assumed to have an autoregressive structure so that each successive period's value is structurally dependent on past values. While this is a step in the 
right direction, it doesn't alleviate the fact that there are many variables on which the ultimate obligation estimate is based. Our approach, on the other hand, abstracts from directly modeling the demographics directly and treats the growth rates of benefits and contributions as returns from a hypothetical security. Using the intuition of BKR, we rewrite the open group unfunded liability as a simple function of the prices of these securities. To value this imaginary asset, we use the notion of arbitrage pricing theory to argue that the financial component of the risk associated with the benefit and contribution cash flows can be replicated using a set of traded assets. We then regress the growth rates over various horizons on the returns of different assets. Using these parameter estimates, we then value the hypothetical growth rate security using asset pricing techniques which have been refined over the past thirty years.

The fruits of our labor are a set of mixed results. For one set of regressors, our estimates of the unfunded liability are on the order of $\$ 58.4$ to $\$ 75.4$ trillion, several times larger than that of the SSA. However, for other regressors, the results show positive as well as negative obligations. When short term and long term government issued securities are added to the regression, the contribution estimates diverge to infinity. These inconsistencies likely reflect estimation off a relatively short data set, which potentially introduces a considerable amount of noise into the parameter estimates. Moreover, it is possible that our assumption that financial markets sufficiently reflect changes in demographics is too strong, which could bias our valuations of the growth rate security prices. A possible topic of future research is to apply this same technique not to the growth rates of benefits and contributions themselves, but rather to the per capita values. This approach would require population projections into the infinite future which adds an additional degree of complexity to the procedure. Nonetheless, the main idea still relies on arbitrage pricing theory to price the per capita growth rates base on marketed asset returns. 


\section{References}

[1] Bakshi, Gurdip S., and Zhiwu Chen. "Baby Boom, Population Aging, and Capital Markets." Journal of Business. Vol. 67, No. 2, (April 1994), p. 165-202.

[2] Bergantino, Steven M. "Life Cycle Investment Behavior, Demographs, and Asset Prices." Unpublished Doctoral Dissertation. Massachusetts Institute of Technology, Economics Department. (Sept. 1998).

[3] Blocker, Alexander W., Laurence J. Kotlikoff, and Stephen A. Ross. "The True Cost of Social Security." NBER Working Paper No. 14427. (March 2009). Available at http://www.nber.org/papers/w14427.

[4] Geanakoplos, John, and Stephen P. Zeldes. "Market Valuation of Accrued Social Security Benefits." NBER Working Paper No. 15170. (July 2009). Available at http://www.nber.org/papers/w15170.

[5] Poterba, James M. "Demographic Structure and Asset Returns." The Review of Economics and Statistics. Vol. 83, No. 4, (Nov 2001), p. 656-684. 


\section{Appendix}

For the regressions with contemporaneous asset returns, the derivation of (13) just requires repeated use of the law of iterated expectations:

$$
\begin{aligned}
G_{y, t, T} & =\mathrm{E}_{t}^{\mathcal{Q}}\left[\prod_{j=1}^{T}\left(\frac{1+g_{y, t+j}}{1+r}\right)\right] \\
& =\mathrm{E}_{t}^{\mathcal{Q}}\left[\prod_{j=1}^{T-1}\left(\frac{1+g_{y, t+j}}{1+r}\right) \mathrm{E}_{t+T-1}^{\mathcal{Q}}\left[\left(\frac{1+g_{y, t+T}}{1+r}\right)\right]\right] \\
& =\left(\frac{1+\alpha+r \sum_{i=1}^{n} \beta_{i}}{1+r}\right) \mathrm{E}_{t}^{\mathcal{Q}}\left[\prod_{j=1}^{T-1}\left(\frac{1+g_{y, t+j}}{1+r}\right)\right] \\
& =\left(\frac{1+\alpha+r \sum_{i=1}^{n} \beta_{i}}{1+r}\right) \mathrm{E}_{t}^{\mathcal{Q}}\left[\prod_{j=1}^{T-2}\left(\frac{1+g_{y, t+j}}{1+r}\right) \mathrm{E}_{t+T-2}^{\mathcal{Q}}\left[\left(\frac{1+g_{y, t+T-1}}{1+r}\right)\right]\right] \\
& =\left(\frac{1+\alpha+r \sum_{i=1}^{n} \beta_{i}}{1+r}\right)^{2} \mathrm{E}_{t}^{\mathcal{Q}}\left[\prod_{j=1}^{T-2}\left(\frac{1+g_{y, t+j}}{1+r}\right)\right] \\
& \vdots \\
& =\left(\frac{1+\alpha+r \sum_{i=1}^{n} \beta_{i}}{1+r}\right)^{T}
\end{aligned}
$$

which shows the result in equation (13).

The price of the $T$-period growth rate security based on the regressions with lagged asset prices, (15), is somewhat more involved. Firstly, note that in the case where $T \leq h$, the market component of all future growth rates is known at the current time $t$ because the furthest growth 
rate from now, $g_{y, t+T}$, is a function of $s_{i, t+T-h}$. Therefore in the case where $T \leq h$, we have

$$
\begin{aligned}
G_{y, t, T} & =\mathrm{E}_{t}^{\mathcal{Q}}\left[\prod_{j=1}^{T}\left(\frac{1+g_{y, t+j}}{1+r}\right)\right] \\
& =\mathrm{E}_{t}^{\mathcal{Q}}\left[\prod_{j=1}^{T}\left(\frac{1+\alpha+\sum_{i=1}^{n} \beta_{i} s_{i, t+j-h}+\epsilon_{t+j}}{1+r}\right)\right] \\
& =\prod_{j=1}^{T} \mathrm{E}_{t}^{\mathcal{Q}}\left[\frac{1+\alpha+\sum_{i=1}^{n} \beta_{i} s_{i, t+j-h}+\epsilon_{t+j}}{1+r}\right] \\
& =\prod_{j=1}^{T}\left(\frac{1+\alpha+\sum_{i=1}^{n} \beta_{i} s_{i, t+j-h}+\mathrm{E}_{t}^{\mathcal{Q}}\left[\epsilon_{t+j}\right]}{1+r}\right] \\
& =\prod_{j=1}^{T}\left(\frac{1+\alpha+\sum_{i=1}^{n} \beta_{i} s_{i, t+j-h}}{1+r}\right)
\end{aligned}
$$

where the third equality follows from the assumption that $\mathrm{E}_{t}^{\mathcal{Q}}\left[\epsilon_{t+j} \epsilon_{t+k}\right]=0$ for any $j$ and $k$.

The case where $T>h$ is derived similarly to the contemporaneous regressions specification. That is, we begin by writing each of the outflow growth rates as functions of the asset returns according to the regression specification:

$$
\begin{aligned}
G_{y, t, T} & =\mathrm{E}_{t}^{\mathcal{Q}}\left[\prod_{j=1}^{T}\left(\frac{1+g_{y, t+j}}{1+r}\right)\right] \\
& =\mathrm{E}_{t}^{\mathcal{Q}}\left[\prod_{j=1}^{T}\left(\frac{1+\alpha+\sum_{i=1}^{n} \beta_{i} s_{i, t+j-h}+\epsilon_{t+j}}{1+r}\right)\right]
\end{aligned}
$$

Notice that the asset returns in the first $h$ terms of the product are known at time $t$. These 
terms will come out of the time $t$ expectation as follows:

$$
\begin{aligned}
G_{y, t, T}= & \mathrm{E}_{t}^{\mathcal{Q}}\left[\prod_{j=1}^{T}\left(\frac{1+\alpha+\sum_{i=1}^{n} \beta_{i} s_{i, t+j-h}+\epsilon_{t+j}}{1+r}\right)\right] \\
= & \mathrm{E}_{t}^{\mathcal{Q}}\left[\prod_{j=1}^{h}\left(\frac{1+\alpha+\sum_{i=1}^{n} \beta_{i} s_{i, t+j-h}+\epsilon_{t+j}}{1+r}\right) \prod_{j=h+1}^{T}\left(\frac{1+\alpha+\sum_{i=1}^{n} \beta_{i} s_{i, t+j-h}+\epsilon_{t+j}}{1+r}\right)\right] \\
= & \prod_{j=1}^{h} \mathrm{E}_{t}^{\mathcal{Q}}\left[\frac{1+\alpha+\sum_{i=1}^{n} \beta_{i} s_{i, t+j-h}+\epsilon_{t+j}}{1+r}\right] \\
& \times \mathrm{E}_{t}^{\mathcal{Q}}\left[\prod_{j=h+1}^{T}\left(\frac{1+\alpha+\sum_{i=1}^{n} \beta_{i} s_{i, t+j-h}+\epsilon_{t+j}}{1+r}\right)\right] \\
= & \prod_{j=1}^{h}\left(\frac{1+\alpha+\sum_{i=1}^{n} \beta_{i} s_{i, t+j-h}}{1+r}\right) \mathrm{E}_{t}^{\mathcal{Q}}\left[\prod_{j=h+1}^{T}\left(\frac{1+\alpha+\sum_{i=1}^{n} \beta_{i} s_{i, t+j-h}+\epsilon_{t+j}}{1+r}\right)\right]
\end{aligned}
$$

where the third equality follows from the assumptions that for each asset $i, \mathrm{E}\left[\epsilon_{t+j} s_{i, t+k}\right]=0$ and $\mathrm{E}\left[\epsilon_{t+j} \epsilon_{t+k}\right]=0$ for any $j$ and $k$. Now we can apply the law of iterated expectations $T-h$ times. The first application gives:

$$
\begin{aligned}
G_{y, t, T}= & \prod_{j=1}^{h}\left(\frac{1+\alpha+\sum_{i=1}^{n} \beta_{i} s_{i, t+j-h}}{1+r}\right) \mathrm{E}_{t}^{\mathcal{Q}}\left[\prod_{j=h+1}^{T-1}\left(\frac{1+\alpha+\sum_{i=1}^{n} \beta_{i} s_{i, t+j-h}+\epsilon_{t+j}}{1+r}\right)\right. \\
& \left.\times \mathrm{E}_{t+T-h-1}^{\mathcal{Q}}\left[\frac{1+\alpha+\sum_{i=1}^{n} \beta_{i} s_{i, t+T-h}+\epsilon_{t+T-h}}{1+r}\right]\right] \\
= & \prod_{j=1}^{h}\left(\frac{1+\alpha+\sum_{i=1}^{n} \beta_{i} s_{i, t+j-h}}{1+r}\right) \mathrm{E}_{t}^{\mathcal{Q}}\left[\prod_{j=h+1}^{T-1}\left(\frac{1+\alpha+\sum_{i=1}^{n} \beta_{i} s_{i, t+j-h}+\epsilon_{t+j}}{1+r}\right)\right. \\
& \left.\times\left(\frac{1+\alpha+\sum_{i=1}^{n} \beta_{i} \mathrm{E}_{t+T-h-1}^{\mathcal{Q}}\left[s_{i, t+T-h}\right]+\mathrm{E}_{t+T-h-1}^{\mathcal{Q}}\left[\epsilon_{t+T-h}\right]}{1+r}\right)\right] \\
= & \left(\frac{1+\alpha+r \sum_{i=1}^{n} \beta_{i}}{1+r}\right) \prod_{j=1}^{h}\left(\frac{1+\alpha+\sum_{i=1}^{n} \beta_{i} s_{i, t+j-h}}{1+r}\right) \\
& \times \mathrm{E}_{t}^{\mathcal{Q}}\left[\prod_{j=h+1}^{T-1}\left(\frac{1+\alpha+\sum_{i=1}^{n} \beta_{i} s_{i, t+j-h}+\epsilon_{t+j}}{1+r}\right)\right]
\end{aligned}
$$


Repeating this procedure $T-h-1$ more times results in:

$$
G_{y, t, T}=\left(\frac{1+\alpha+r \sum_{i=1}^{n} \beta_{i}}{1+r}\right)^{T-h} \cdot \prod_{j=1}^{h}\left(\frac{1+\alpha+\sum_{i=1}^{n} \beta_{i} s_{i, t+j-h}}{1+r}\right)
$$

Combining the results for $T>h$ with those for $T \leq h$ confirms equation (15):

$$
G_{y, t, T}=\left(\frac{1+\alpha+r \sum_{i=1}^{n} \beta_{i}}{1+r}\right)^{\max (T-h, 0)} \cdot \prod_{j=1}^{\min (h, T)}\left(\frac{1+\alpha+\sum_{i=1}^{n} \beta_{i} s_{i, t+j-h}}{1+r}\right)
$$

